We are also grateful to Dr. G. V. D. Tiers for several stimulating discussions.

Note added in proof. After this paper had been submitted, Dr. John Foss (University of Oregon) directed our attention to a paper by Sverdlova ${ }^{13}$ in which similar data are presented for benzene and chlorobenzene. In general, these data agree with ours ; but they are plotted in such a way as to obscure the disagreement with theoretical prediction.

F. A. Bovey

S. S. YANARI

Central Research Laboratories,

Minnesota Mining and Manufacturing Co., St. Paul, Minnesota.

1 Bayliss, N. S., J. Chem. Phys., 18, 292 (1950).

'Longuet-Higgins, H. E., and Pople, J. A., J. Chem. Phys., 27, 192 (1957).

${ }^{3}$ Ooshika, Y., J. Phys. Soc. Japan, 9, 594 (1954).

4 McRae, E. G., J. Phys. Chem., 61, 562 (1957).

${ }^{5}$ Lauer, K., and Oda, R., Ber., 69, 851 (1936).

'Shepard, S. E., Rev. Mod. Phys., 14, 303 (1942).

${ }^{7}$ Phibbs, M. K., J. Chem. Phys., 19, 1430 (1951).

${ }^{8}$ Ham, J., J. Amer. Chem. Sox., 76, 3881 (1954)

- Yanari, S. S., and Bovey, F. A., J. Biol. Chem. (in the press)

${ }^{10}$ Matsen, F. A., Ginsberg, N., and Robertson, W. W., J. Chem. Phys, 13, $309(1945)$

${ }^{11}$ Brice, T. J., in "Fluorine Chemistry", edit. by Simons, J. H., 436 (Academic Press, New York, 1950).

${ }^{12}$ Simons, J. H, and Dunlay, J. Chem. Phys., 18, 345 (1950).

${ }^{13}$ Sverdlova, O., Optics and Spectroscopy (U.S.S.R.), B, 223 (1959).

\section{Absorption Spectra of Diatomic Radicals containing Boron or Silicon}

VARIous workers have used methods of timeresolved absorption spectroscopy to study the formation and decay of free radicals produced in pulsed electric discharges. These investigations have been confined to the use of a single parent substance which normally flows through the discharge tube at a rate such that the parent substance is not completely replenished before each discharge pulse. In this way the absorption spectra of $\mathrm{OH}$ (ref. 1), $\mathrm{CN}$ (ref. 2) and of several halides such as $\mathrm{BBr}, \mathrm{BCl}$, AlBr, AlCl, AlI and SiCl ${ }^{3,4}$ have been observed. The main limitation in this work was low intensity of the background sources used, and a large number of exposures were needed to obtain an adequate record with both high dispersion and good time resolution. The use of a flash tube filled with an inert gas as background source greatly reduces the number of exposures needed to obtain an adequate record and this removes the difficulty of studying systems where the diseharge tube has to be refilled after each exposure.

An apparatus of this type has been constructed, and has been used for studying the absorption spectra of free radicals which have not previously been detected in absorption and which would be expected to have appreciable concentrations at the temperatures reached when a pulse electric discharge is passed through a suitable gas mixture at low pressure. In typical experiments a $1-5 \mu \mathrm{F}$. condenser at $10 \mathrm{kV}$. is discharged between tungsten electrodes $25 \mathrm{~cm}$. apart in a $5-\mathrm{cm}$. diameter glass tube containing the parent mixture at a few $\mathrm{mm}$. mercury pressure. A $100 J$ argon-filled quartz capillary flash tube with a duration of 7 usec. is used to photograph the absorption spectrum on a 3-metre grating spectrograph. The firing of this lamp is controlled by an electronic delay circuit which is initiated by light from the discharge. With the optical arrangement used, the amount of light reaching the spectrograph from the discharge is small enough by comparison with intensity of the spectroscopic flash to make the use of shuttering unnecessary at wave-lengths above $2800 \AA$.

To date experiments have been concentrated on systems containing boron or silicon. For the former, either boron trichloride or trimethyl borate has been used, either alone or mixed with oxygen, nitrogen or hydrogen. In these systems, the absorption spectra of $\mathrm{BCl}\left(A^{1} \Pi I-X^{1} \Sigma\right), \mathrm{BH}\left(A^{1} \Pi\left[-X^{1} \Sigma^{+}\right), \mathrm{BN}\left({ }^{3} \Pi-{ }^{3} \Pi\right)\right.$, $\mathrm{BO}\left(A^{2} \Pi-X^{2} \Sigma^{+}\right)^{5}$ and the "boric acid fluctuation bands"" have been observed. Of these, the observation of the triplet but not the singlet bands of $B N$ (ref. 7) in absorption is of particular interest, since they suggest strongly that $\mathrm{BN}$ has a ${ }^{3} \mathrm{il}$ ground-state, whereas the iso-electronic species $\mathrm{C}_{2}$ has recently been shown to have a ${ }^{1} \Sigma^{+}$ground-state with its configurationally excited ${ }^{3} \Pi$ state only about $610 \mathrm{~cm} .^{-1}$ higher ${ }^{8}$. This difference in behaviour can be attributed to the fact that ground-state $\mathrm{B}\left({ }^{2} P\right)$ and $\mathrm{N}\left({ }^{4} S\right)$ atoms can only give triplet and quintet states, whereas singlet, triplet and quintet states can arise from two normal carbon atoms $\left({ }^{3} P\right)$. Attempts are being made to observe the gas-phase absorption spectrum of $\mathrm{BeO}$ which is iso-electronic with $\mathrm{BN}$ and $\mathrm{C}_{2}$ with the view of determining the relative positions of its lowest singlet and triplet states.

Preliminary oxperiments with silicon tetrachloride either alone or mixed with hydrogen or nitrogen have yielded the absorption spectra of SiCl $\left(B^{2} \Sigma-X^{2} \mathrm{II}\right)$, SiH $\left(A^{2} \Delta-X^{2} 11\right)$ and $\operatorname{SiN}\left(B^{2} \Sigma^{+} \cdots X^{2} \Sigma^{+}\right)^{5}$. In addition to observing most of the $B^{2} \Sigma-X^{2} 11$ bands of SiCl listed by Jevons', the $(2,1)$ and $(3,1)$ bands have been identified. The identification of these bands and the strong appearance of the $(2,0)$ and $(3,0)$ bands in these experiments confirm Jevons's assignments, about which he expressed some doubt. Under conditions when the ${ }^{2} \Delta-{ }^{2} \Pi$ band of SiH was most intense, a weak, slightly diffuse system appeared in absorption between 3900 and $3750 \AA$. This spectrum has been tentatively identified as the ${ }^{2} \Sigma-{ }^{2}$ il transition of $\mathrm{SiH}$ to be expected in this region by analogy with CH. An attempt is boing made to obtain this spectrum with enough intensity to permit a rotational analysis. No band system of $\mathrm{SiH}$ corresponding to the $3143 \AA$ A. ${ }^{2} \Sigma^{+}-{ }^{2} I I$ transition of $\mathrm{CH}$ has yet been detected.

It can be seen that this technique provides a relatively simple method of studying the absorption spectra of species likely to be present at high temperatures without the use of large quantities of material or of elaborate apparatus to produce the high temperatures. A detailed account of this work is being prepared for publication.

I wish to thank Prof. R. G. W. Norrish for helpful discussions and the Government Grants Committee of the Royal Society for a grant, part of which was applied to this work.

Department of Physical Chemistry,

$$
\text { B. A. Thrush }
$$

'Oldenberg, O., J. Phys. Chem., 41, 293 (1937).

2 White, J. U., J. Chem, Phys., 8, 79, 459 (1940).

${ }_{3}$ Miegcher, E. Helv. Phys, Acta, 9, 693 (1936).

- Maeder, D., Helv. Phys. Acta, 16, 503 (1943).

"Maeder, D., Helv. Phys. Acta, 16, second ed. (Van Herzberg, G., "Spectra of Dia
Nostrand, New York, 1950).

6 Jevons, W., Proc. Roy. Soc., A, 81, 120 (1915).

$\checkmark$ Douglas, A. E., and Herzberg, G., Canad. J. Res., A, 18, 179 (1940).

s Ballik, E. A and Ramsay, D. A., J. Chem. Phys., 31, 1128 (1959).

- Jevons, W., Proc. Phys. Soc., 48, 563 (1936). 